\title{
Fibrocystic liver disease: novel concepts and translational perspectives
}

\author{
Alberto Lasagni ${ }^{1}$, Massimiliano Cadamuro ${ }^{1}$, Giovanni Morana ${ }^{2}$, Luca Fabris ${ }^{1,3}$, Mario Strazzabosco ${ }^{3}$ \\ ${ }^{1}$ Department of Molecular Medicine, University of Padua, Padua, Italy; ${ }^{2}$ Division of Radiology, Treviso Regional Hospital, Treviso, Italy; ${ }^{3}$ Liver \\ Center and Section of Digestive Diseases, Department of Internal Medicine, Yale University School of Medicine, New Haven, CT, USA \\ Contributions: (I) Conception and design: L Fabris, M Strazzabosco; (II) Administrative support: A Lasagni; (III) Provision of study materials or \\ patients: None; (IV) Collection and assembly of data: None; (V) Data analysis and interpretation: All authors; (VI) Manuscript writing: All authors; \\ (VII) Final approval of manuscript: All authors. \\ Correspondence to: Luca Fabris, MD, PhD. Department of Molecular Medicine (DMM), University of Padua, Via A. Gabelli 63, Padua 35121, Italy. \\ Email: luca.fabris@unipd.it.
}

\begin{abstract}
Fibrocystic liver diseases (FLDs) comprise a heterogeneous group of rare diseases of the biliary tree, having in common an abnormal development of the embryonic ductal plate caused by geneticallydetermined dysfunctions of proteins expressed in the primary cilia of cholangiocytes (and therefore grouped among the "ciliopathies"). The ductal dysgenesis may affect the biliary system at multiple levels, from the small intrahepatic bile ducts [congenital hepatic fibrosis (CHF)], to the larger intrahepatic bile ducts [Caroli disease (CD), or Caroli syndrome (CS), when CD coexists with $\mathrm{CHF}$, leading to biliary microhamartomas and segmental bile duct dilations. Biliary changes are accompanied by progressive deposition of abundant peribiliary fibrosis. Peribiliary fibrosis and biliary cysts are the fundamental lesions of FLDs and are responsible for the main clinical manifestations, such as portal hypertension, recurrent cholangitis, cholestasis, sepsis and eventually cholangiocarcinoma. Furthermore, FLDs often associate with a spectrum of disorders affecting primarily the kidney. Among them, the autosomal recessive polycystic kidney disease (ARPKD) is the most frequent, and the renal function impairment is central in disease progression. $\mathrm{CHF}$, $\mathrm{CD} / \mathrm{CS}$, and ARPKD are caused by a number of mutations in polycystic kidney hepatic disease 1 (PKHD1), a gene that encodes for fibrocystin/polyductin, a protein of unclear function, but supposedly involved in planar cell polarity and other fundamental cell functions. Targeted medical therapy is not available yet and thus the current treatment aims at controlling the complications. Interventional radiology or surgical treatments, including liver transplantation, are used in selected cases.
\end{abstract}

Keywords: Fibrocystic liver disease (FLD); congenital hepatic fibrosis (CHF); Caroli disease (CD); Caroli syndrome (CS); biliary fibrosis; polycystic kidney hepatic disease 1 (PKHD1)

Received: 14 April 2020. Accepted: 05 June 2020; Published: 05 April 2021.

doi: 10.21037/tgh-2020-04

View this article at: http://dx.doi.org/10.21037/tgh-2020-04

\section{Introduction}

Fibrocystic liver diseases (FLDs) designate a group of rare disorders affecting the biliary tree, characterized by an aberrant morphogenesis of the bile ducts, resulting in segmental dilations, eventually associated to cysts in other organs. Common traits of FLDs are the rare incidence, the congenital origin, and the unique pathogenesis related to an abnormal development of the ductal plate (the embryonic structure originating the intrahepatic bile ducts). Ductal plate malformations (DPMs) encompass von Meyenburg complex (VMC), congenital hepatic fibrosis (CHF), Caroli disease (CD) and Caroli syndrome (CS), and choledochal cysts (CCs). Schematically, each FLD involves distinct anatomical levels, as outlined in Table 1 (1). However, in reality, the demarcation between these conditions is not 
Table 1 FLD classification according to the level of biliary tree involvement

\begin{tabular}{llc}
\hline Disease & \multicolumn{1}{c}{ Biliary tree level involved } & Size \\
\hline VMC & Small intralobular bile ducts & $<20 \mu \mathrm{m}$ \\
CHF & Interlobular and septal bile ducts & $20-50 \mu \mathrm{m}$ \\
$\begin{array}{l}\text { Caroli's } \\
\text { disease }\end{array}$ & Larger intrahepatic bile ducts & $>50 \mu \mathrm{m}$ \\
$\begin{array}{l}\text { Caroli's } \\
\text { syndrome }\end{array}$ & From interlobular to larger intrahepatic & $>20 \mu \mathrm{m}$ \\
$\begin{array}{l}\text { Choledochal ducts (CHF + CD) } \\
\text { cysts }\end{array}$ & Extrahepatic bile ducts & $2-8 \mathrm{~mm}$ \\
\hline
\end{tabular}

clear, as the intrahepatic biliary tree can be simultaneously affected at multiple levels. For example in CS, large bile duct ectasia coexists with $\mathrm{CHF}$, a condition typically affecting the smaller bile ducts. Moreover, biliary changes can be associated to cysts developing in other ductal epithelia, more often renal [autosomal recessive polycystic kidney disease (ARPKD)], but also pancreatic. Not rarely, patients show a combination of renal and hepatic changes, with CHF and CS coexisting with ARPKD. FLDs belong to a much wider group of developmental diseases called "ciliopathies", to highlight the notion that dysfunction of the cilium plays a key role in their pathogenesis $(2,3)$.

\section{Ciliopathies}

Cellular cilia are categorized as motile or non-motile. Motile cilia are expressed by the respiratory, fallopian tube, sperm and ependymal epithelial cells, and are involved in the regulation of fluid transport across the epithelial surfaces. Their dysfunction is associated with a variety of conditions, including bronchiectasis, situs viscerum inversus, and infertility $(4,5)$. Non-motile cilia are sensory organelles expressed by most polarized eukaryotic cells, including cholangiocytes and renal tubular epithelial cells. They lay on basal bodies (centrioles) and extend outward from the cell surface to serve as signal transducers between extracellular fluids (e.g., urine, bile) and the intracellular environment (6). Upon entry in the cell cycle, non-motile cilia are disassembled, leaving the basal bodies free to arrange the mitotic spindle that will drive separation of chromosomes. Non-motile cilia harbour a group of proteins (including polycystins, fibrocystin, polaris) mediating cellcell and cell-matrix interactions that are crucial for tissue development, regeneration/repair and homeostasis (7).
Ciliopathies caused by defects in non-motile ciliary proteins are characterized by a wide degree of ductal dysgenesis that may result in the development of cystic lesions. For instance, in ciliopathies targeting the kidneys, clinical manifestations can range from mild urinary concentration defects in normal appearing kidneys, to kidney with a clear abnormal morphology and severe functional impairment. The most common renal ciliopathies are autosomal dominant and recessive polycystic kidneys disease (ADPKD and ARPKD), but nephronophthisis, cystic dysplastic kidneys, medullary sponge kidney and various overlap syndromes are also worth mentioning (2). Within ciliopathies with hepatic involvement, FLDs are distinct from polycystic liver diseases (autosomal dominant polycystic liver disease, ADPLD and autosomal dominant polycystic kidney disease, ADPKD) on genetic, pathophysiological, and clinical grounds.

\section{Genetics}

Genetic defects responsible for ciliopathies have started to be elucidated in the early 90s. Polycystic kidney hepatic disease 1 (PKHD1) is the most frequently involved gene in FLDs, such as CHF/CD and ARPKD. PKHD1 is a complex gene of about $500 \mathrm{~kb}$, located on the chromosome $6 \mathrm{p} 21.1 \mathrm{p} 12$ encoding for fibrocystin/polyductin (FPC). Genetic structure of PKHD1 has been analysed leading to the identification of over 300 mutations, with a detection rate ranging from $42 \%$ to $87 \%$ (8). However, the overall picture is even more complex, as clinical features and progression rate of renal or hepatic disease are independent and may vary within a given PKHD1 mutation, suggesting the intervention of other unknown phenotype modifying genes (9).

Missense, deletion/insertion, and splicing mutations have been described in ARPKD patients. The most frequent pathogenic variants of PKHD1 gene are nonsense truncating mutations (around $60 \%$ ), while missense mutations account for $40 \%$ and in particular, the most common missense mutation of this gene, the c. $107 \mathrm{C}>\mathrm{T}$ (p.Thr36Met), has been estimated to affect the $20 \%$ of all patients. Moreover, regarding PKHD1 mutations, 33\% are present in a single family, whilst only $10-20 \%$ are common to PKHD1 defective patients (10-12). Given the high frequency of missense mutations, in particular single nucleotide mutations, an ARPKD mutation database has been created to support genetic studies and interpretation of genetic testing in view to predict the severity of the 
Table 2 Genetics of FLD-related syndromes

\begin{tabular}{lccl}
\hline Mutated gene & Liver phenotype & Associated syndrome & \multicolumn{1}{c}{ Clinical features } \\
\hline PKHD1 & CHF, CD & ARPDK & Renal collecting duct dilation \\
PKD1-2 & CHF, biliary cysts & ADPDK & Nephron, pancreatic, aracnoidal, seminal vesicles cysts \\
NPHP1-15 & CHF & NPHP & $\begin{array}{l}\text { Fibrosis at the tubule-interstitial level, cysts at cortico-medullar junction, retinal } \\
\text { degeneration, situs inversus }\end{array}$ \\
JBTS1-20 & CHF, CD & Joubert & Renal cystic dysplasia, hypo/aplasia of cerebelar vermis \\
BBS1-15 & CHF & Bardet-Biedl & Renal cystic dysplasia, obesity, intellectual disability, hypogonadism \\
MKS1-10 & CHF & Meckel-Gruber & Renal cystic dysplasia, polydactyly; high perinatal mortality \\
OFD1 & CHF & Oral-Facial-Digital 1 & Glomerular cysts, face malformation, occipital omphalocele \\
ATD1-5 & CHF, CD & Jeune & $\begin{array}{l}\text { Renal cystic dysplasia, short stature, skeletal dysplasia, polydactyly; high } \\
\text { perinatal mortality }\end{array}$ \\
\hline
\end{tabular}

Adapted from (15).

disease (13). Multiple studies have suggested the presence of genotype-phenotype correlations. Indeed, most of the ARPKD patients with severe phenotypes present associations to several single missense-truncating mutations in homozygous form, whereas subjects harbouring two truncating mutations do not survive the neonatal age. Moreover, mutation detection rates are higher for patients with severe, early-onset disease, because they usually show truncating mutations that are easier to recognize $(12,14,15)$.

The protein product of PKHD1 is fibrocystin/polyductin (FPC), a receptor-like protein localized in the basal body of cilia and centromeres, and in cell-cell junctions, predominantly in collecting ducts and thick ascending loop epithelium in the kidney, and in ductal epithelium of liver and pancreas. FPC function is yet far to be deciphered, but it is likely involved in multiple cell activities, as confirmed by the pre-natal and peri-natal disease manifestations that result when it is mutated. Its action is linked to proliferation, secretion, terminal differentiation, and heterotypic interactions with the extracellular matrix (16). Moreover, FPC is functionally active in adult tissues suggesting a range of post-developmental roles. Indeed, PKHD1 encodes membrane-bound and soluble FPC isoforms that can be detected at distinct subcellular localizations. Moreover, its localization at the epithelial basal bodies/cilia overlaps the expression of polycystin (PC) 1 and 2, both mutated proteins in ADPKD with cystic liver involvement, meaning that PKHD1 may physically interact and cooperate with PC1 and PC2 in driving common pathways that modulate ciliary function and cation-channel activity. Furthermore, FPC may undergo a Notch-like processing with a controlled release of the ectodomain portion from the primary cilia suggesting a novel mechanism whereby ciliary proteins may behave as bi-directional signalling molecules (16). The evidence of cytoplasmic staining for FPC support an additional binding role for a group of FPC isoforms, whose effects are mostly unexplored. That said, the final effect of FPC is the result of the multiple actions exerted by the different isoforms, dependent upon tissue localization, functional contexts, and stages of organ development $(17,18)$.

Besides PKHD1, other genes can be involved in the pathogenesis of FLD, and they are reported in Table 2. Among them, mutations in IFT88/Polaris have gained attention as experimental model of CHF. IFT88/Polaris encodes a component of the intracellular transport system, involved in cell cycle and ciliogenesis, and mice with IFT88/ Polaris inactivation develops a liver phenotype similar to FPC deficiency (19).

\section{Embryology}

A key feature of the hepatic phenotype in hepatic ciliopathies is an altered morphogenesis of the biliary tree, leading to a typical pathological aspect called DPM $(20,21)$. The biliary system starts to develop at the $8^{\text {th }}$ week of gestation from the endodermal hepatic diverticulum of the ventral foregut endoderm. The intrahepatic bile duct epithelium originates from the cranial part, while the extrahepatic portion of the biliary tree derives from the caudal part of the ventral foregut endoderm. In the liver parenchyma, the primordial biliary structure is the "ductal plate", a single layer of hepatoblasts with bipotential 


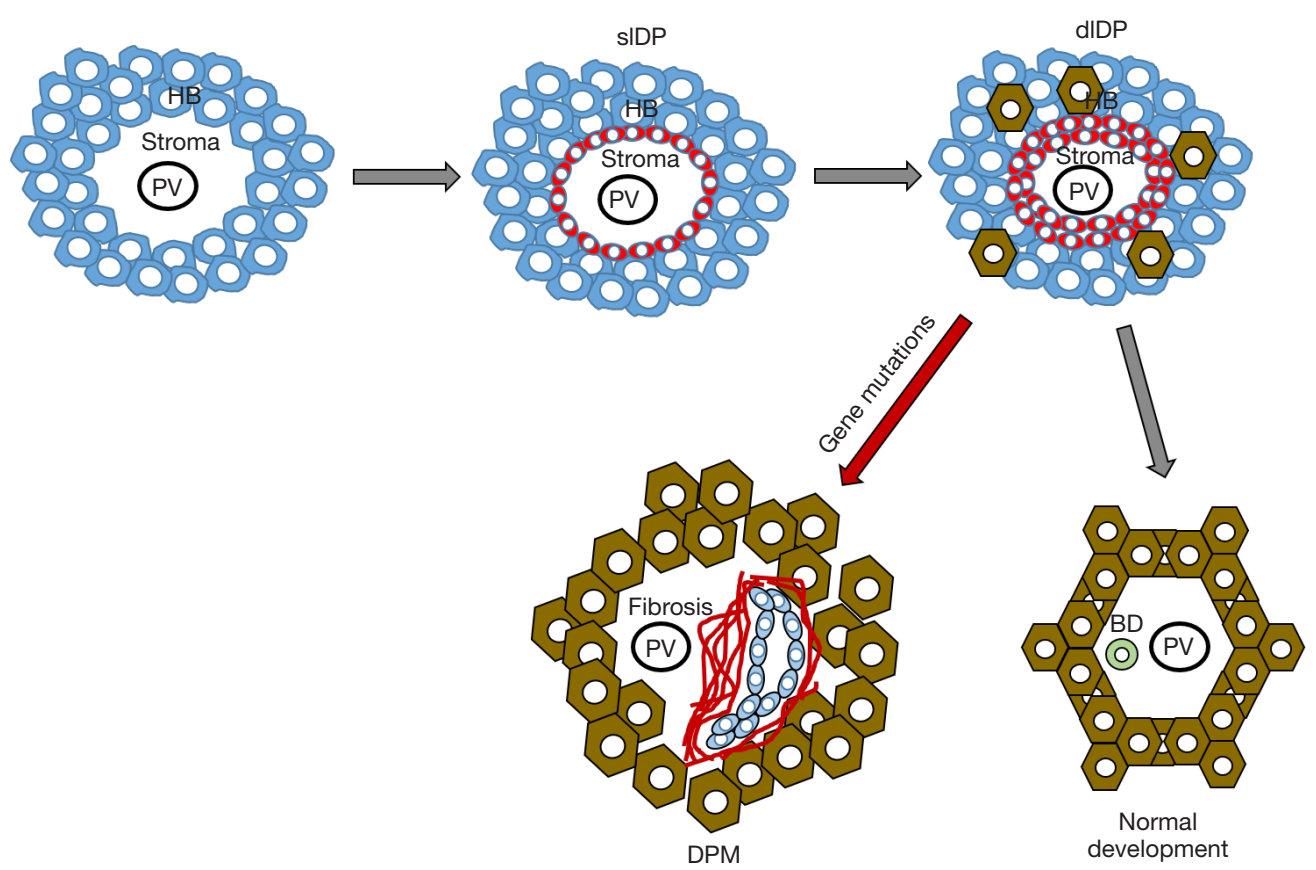

Figure 1 Fetal development of intrahepatic bile duct epithelium is a highly coordinated process involving several different cell types. Initially, the hepatoblasts (HB) aligning along the nascent portal tract centered around the portal vein (PV) branches are primed to gain biliary-like characteristics and transdifferentiate into single layer ductal plate (slDP, red cells), the primordial structure of the bile duct, that further progresses to double layer ductal plate (dIDP). In normal conditions, dlDP migrate into the portal mesenchyme to become incorporated as fully differentiated bile ducts (BD). When this maturation process is perturbed, as by genetic defects, DPM develop at the margins of the portal tract, and are often associated with the nearby deposition of fibrotic tissue.

capabilities differentiating from those localized in the area abutting the nascent portal area. Ductal plates evolve to double layered structures enclosing a primitive lumen; the layer facing the portal mesenchyme will originate the biliary epithelium, whereas the opposite layer will originate hepatocytes. After the "duplication" stage, with progressive dilation of the lumen, tubular structures migrate into the portal mesenchyme whereby they are incorporated. Once the lumen is created, around the $30^{\text {th }}$ week intrahepatic bile ducts mature along cross-sectional and cranio-caudal axis directed from the hilum to the periphery. Progressive elongation of the bile ducts is critically regulated by an intricate mechanism that orientates mitosis along the right axis and maintains the tubular architecture within the ductal plane, the so-called "planar cell polarity" (PCP). This process is directed by mutual interactions between ductal plate and mesenchymal cells under the control of a huge number of growth and transcription factors, stimulating cell migration and cholangiocyte differentiation. If defective, this mechanism leads to abnormal dilated bile ducts resulting into biliary cystic or "cyst-like" lesions $(20,22)$.
The different steps of the foetal development of intrahepatic bile duct epithelium are exemplified in Figure 1.

\section{Molecular pathogenesis}

Experimental models of nephron postnatal morphogenesis showed a clear correlation between renal cystogenesis and loss of planar cell polarity (PCP) (23), a signalling mechanism establishing and maintaining the planar configuration of renal tubules during development (24). Moreover, after development, the primary cilia-basal body-centrosomal complex regulates pathways that determine the planar orientation of individual cells within the architecture of the ducts. In this regard, the function of the primary cilia has been linked to non-canonical Wnt signalling (25). Notably, in two toxicant models of chronic cholangiopathies (the 3,5-diethoxycarbonyl1,4-dihydrocollidine and the thioacetamide-treated mice), activation of the non-canonical Wnt signalling in cholangiocytes by $\mathrm{Wnt} 5 \mathrm{a}$, is responsible for the disruption of PCP in the biliary epithelium. This mechanism is 
associated with increased ductular reaction and accumulation of portal myofibroblasts leading to progressive deposition of collagen typical of fibrosing cholangiopathies (26). The role of non-canonical Wnt and altered PCP in CHF and CS is still uncharted. Other molecular mechanisms can be involved in PCP. Studies in Zebrafish demonstrate that PCP is perturbed by knockdown of Scribble, a protein belonging to the polarity complex, expressed on the cell-cell junctions, that leads to formation of cyst-like structures in the kidney $(27,28)$. Whether alterations in Scribble might contribute to biliary dysgenesis in PKHD1 mutations has not been investigated.

In addition to the morphogenetic role supporting PCP, FPC exerts an inhibitory tone on a latent, pro-inflammatory phenotype displayed by the biliary epithelium, which is reminiscent of a developmental behaviour, necessary to undertake the extensive web of cell communications occurring in embryogenesis. When FPC is defective, $\beta$-catenin signaling is overactivated, leading to increased secretion of cyto/chemokines (such as CXCL1, CXCL10, CXCL12) that are able to attract macrophages and mesenchymal cells in the peribiliary area, ultimately resulting in a progressive collagen deposition around the dysgenetic ducts. In FPC-defective cholangiocytes, chemokine secretion may be further enhanced by a local, self-perpetuating feed-forward loop sustained by IL$1 \beta$ through the activation of the JAK-signal transducer and activator of transcription 3 (STAT3) pathway, which operates through an activated inflammasome (29-31).

Activated myofibroblasts are the main cell type responsible for the generation of extracellular matrix components, and derive from both hepatic stellate cells (HSCs) and portal fibroblasts (PFs). Connective tissue growth factor (CTGF) is another signal released by multiple inflammatory cell types able to promote recruitment and activation of HSCs and PFs (32). Once activated, portal myofibroblasts can also stimulate cholangiocyte proliferation through P2Y receptors, resulting in a feed-forward loop further sustaining PF proliferation and transdifferentiation mediated by the cholangiocyte production of monocyte chemoattractant protein-1 (MCP-1). MCP-1 secretion is possibly stimulated by the increased mechanical stiffness due to cyst growth and fibrotic tissue deposition, thus representing a possible link between cystogenesis and fibrogenesis (33). Importantly, increased expression of genes regulating the innate immune response, including activated complement protein-3 (C3a) and MCP1 receptor, has been reported in $c p k$ mice, an experimental model of ARPKD (34).

Altogether, several soluble factors unleashed by PKHD1 defects have pathogenetic significance in CHF/CS. Starting from the observation that growing biliary cysts are surrounded by a rich innate immune infiltrate and dense collagen deposition, an intriguing speculation suggests these lesions are sustained by tightly interrelated processes, that includes PCP perturbation, cholangiocyte proliferation, inflammation and fibrosis, as depicted in Figure 2 (33). In the context of this triad, there is an overlap of proteins and signalling pathways, involving common cell responses, with strong pro-oncogenic potential. This is an area deserving consideration by future studies with possible traslability to more common acquired chronic liver diseases (29).

\section{Clinical aspects}

Because of their frequent overlap, we will discuss the clinical aspects of CHF, CD and CS together, highlighting the differences.

$\mathrm{CHF}$ is a rare autosomal recessive disease. DPM affects the interlobular bile ducts and is characterized by progressive peribiliary fibrosis leading to portal hypertension and variceal bleeding. Although epidemiological data on the prevalence of $\mathrm{CHF}$ and $\mathrm{CD} / \mathrm{CS}$ are lacking, conditions associated with $\mathrm{CHF}$ seem to affect around 1:10-20,000 subjects, whereas CD/CS is considered even rarer, affecting around 1:1,000,000 subjects. The natural history of $\mathrm{CHF}$ is variable as the severity of clinical manifestations depends not only on portal hypertension, but also on the renal function impairment, given the close association of CHF with ARPKD. Clinical onset is highly variable, ranging from childhood to the $6^{\text {th }}$ decade, though diagnosis is mainly performed in adolescence or young adulthood. However, since initial manifestations are nonspecific, the diagnosis may be deferred until the appearance of complications. Most patients are asymptomatic, while some can complain of mild right upper quadrant abdominal pain eventually accompanied by hepato-splenomegaly (15). At the biochemical level, liver function is usually preserved. Mild elevation of liver enzymes can be observed, but marked cholestasis is rare, occurring in cholangitic forms, more often associated with CS. As shown in Table 3, CHF can be classified in four clinical types according to the predominance of portal hypertension and/or cholestasis.

In CD, DPMs extend beyond the small interlobular bile ducts, to affect the larger intrahepatic bile ducts or even the segmental portions of a single lobe, usually the left one, 


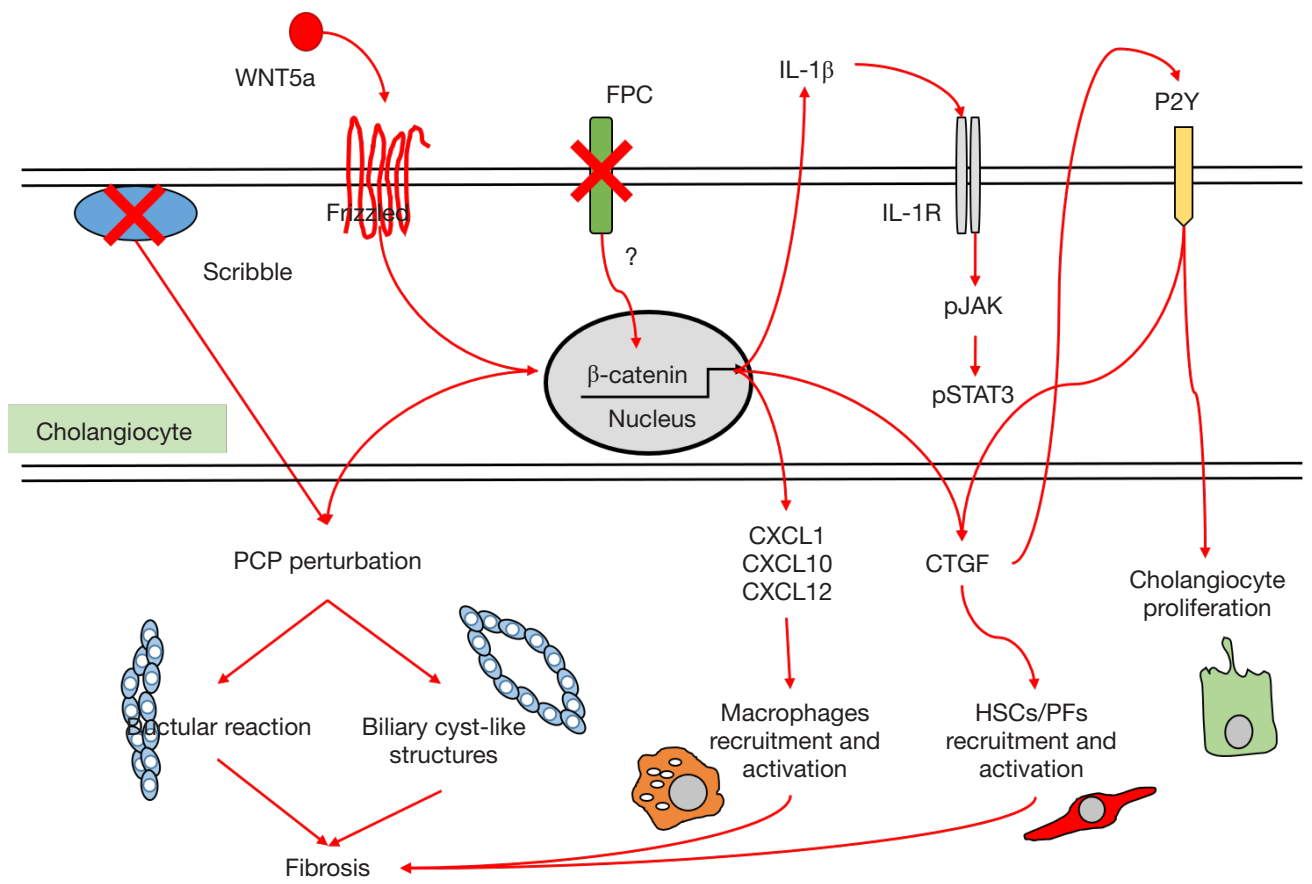

Figure 2 Main molecular mechanisms contributing to cyst development, which act through deregulation of planar cell polarity, stimulation of cholangiocyte proliferation and activation of inflammatory cells and myofibroblasts in the peribiliary area. They involve a complex interplay of numerous cytokines, chemokines, growth factors and morphogens, regulated by $\beta$-catenin and pJAK/STAT3 signaling. Of note, perturbation of planar cell polarity may lead to ductular reaction or cyst-like structure formation, accompanied by progressive peribiliary fibrosis. See text for details. FPC, fibrocystin/polyductin; PCP, planar cell polarity; CTGF, connective tissue growth factor; HSCs, hepatic stellate cells; PFs, portal fibroblasts.

Table 3 Clinical phenotypes of CHF/CS/CD

\begin{tabular}{ll}
\hline Phenotype & \multicolumn{1}{c}{ Clinical features } \\
\hline Portal hypertension* & $\begin{array}{l}\text { Variceal bleeding, hepatosplenomegaly, } \\
\text { thrombocytopenia }\end{array}$ \\
Cholestasis $^{\#}$ & $\begin{array}{l}\text { Recurrent cholangitis, sepsis, pruritus, } \\
\text { intrahepatic lithiasis }\end{array}$ \\
Mixed & $\begin{array}{l}\text { Both portal hypertension and cholestasis } \\
\text { phenotypes }\end{array}$ \\
Latent & Late-onset presentation \\
\hline
\end{tabular}

*, most common form; ", usually associated to CS.

or more rarely, the whole biliary tree as a bi-lobar disease. The resulting bile duct ectasias can be recognized by imaging studies to support early detection. CD is sporadic and less common than CS, which is inherited as autosomal recessive disease, and as $\mathrm{CHF}$, is frequently associated with kidney disease. In $\mathrm{CD}$, clinical course is usually oligo or asymptomatic for all lifelong. As CHF, onset occurs in childhood or teenage, but it is often diagnosed many years later as well, in the fifth decade. The 'biliary cysts' are in direct connection with the biliary tree and so biliary stasis may favour infections and the formation of intrahepatic stones. Symptoms are mostly related to complications, such as acute bacterial cholangitis. Furthermore, recurrent cholangitic episodes can evolve to secondary biliary cirrhosis $(35,36)$. At younger ages, symptoms are more likely related to portal hypertension due to concomitant $\mathrm{CHF}$ in the context of CS.

The main determinants of clinical progression of $\mathrm{CHF}$ and $\mathrm{CD} / \mathrm{CS}$ are portal hypertension, recurrent acute cholangitis and cholangiocarcinoma (CCA). Ascites is uncommon in these patients and the most frequent complications of portal hypertension is variceal bleeding, often the first manifestation of CHF at any age, or splenomegaly with thrombocytopenia. Development of portal vein thrombosis and cavernoma has been reported. The management of portal hypertension does not differ from that of other aetiologies and follows the AASLD/ 
EASL guidelines (37).

Acute cholangitis is more typical of $\mathrm{CD}$ but it may be a lifethreatening complication also in $\mathrm{CHF}$ for the high risk of biliary sepsis. It is generally caused by bacterial infections sustained by Gram-negative Enterobacteria (E. Coli, K. Pneumoniae, Enterobacter spp) (38), and must be suspected in case of fever that could be the only sign of disease in these patients.

Intrahepatic cholangiocarcinoma (iCCA) is a feared complication of $\mathrm{CD} / \mathrm{CS}$ and sometimes of CHF. CCA is likely the result of chronic inflammation and of the progressive fibrosis developing in close vicinity of dysgenetic biliary structures, as observed in other inflammatory cholangiopathies, particularly in primary sclerosing cholangitis (PSC) (39). In CD/CS, it is often diagnosed incidentally at the time of liver surgery. Incidence ranges from 2.5 to $16 \%$ with a median age at diagnosis of 58.8 years (40). Unfortunately, despite remarkable improvements in the radiological approach, no surveillance guidelines for CCA are available so far in these patients.

\section{Associated diseases}

Extrahepatic manifestations of FLDs include cystic dysplastic kidney degeneration, pancreatic cysts, polydactyly, mid and hindbrain abnormalities, retinal degeneration, and iris or retinal colobomas. Among them, ARPKD is the most frequent associated disease as well as the most common ciliopathy in childhood with a incidence of 1:20,000 live births (9,41-43). Similar to FLD, the genetic defect is mainly related to mutations in the PDKHD1 gene. ARPKD is characterized by non-obstructive fusiform dilations of the renal collecting ducts with progressive renal insufficiency. In about $40 \%$ of patients, liver and renal diseases coexist, and thus they are collectively termed hepato-renal fibrocystic disease (HRFCD). However, it is still unclear if severity of both diseases correlates (44). Prognosis is poor with about $30 \%$ of affected infants dying during the neonatal period due to concomitant pulmonary hypoplasia reflecting a generalized multiorgan dysgenesis $(43,45)$. Nevertheless, in the last decade, thanks to the constant improvements in neonatal care, the 10 -year survival has risen up to $80 \%$ of patients and a large percent of survivors will develop liver complications $(43,45)$.

\section{Diagnosis}

The mainstay of diagnosis is radiology. Colour-Doppler liver and kidney ultrasound (US) is the first step in the diagnostic work-up of both liver and kidney disease and their related complications. Typical US findings in FLDs are: (I) increased or heterogeneous liver echogenicity with hyperechoic portal triad and periportal thickening in cases with acute cholangitis; (II) hypertrophy of left lateral and caudate segment, normal left median segment and atrophic right lobe in cases with advanced fibrosis; (III) splenomegaly if portal hypertension; (IV) dilated intrahepatic bile ducts eventually hosting stones in CD. Second-line imaging studies as contrast-enhanced computed tomography (CT) scan and magnetic resonance imaging (MRI) coupled with MR cholangiopancreatography (MRCP) allow a better visualization of the vasculature and biliary tree, respectively, as well as provide a better staging of fibrosis. At imaging, a pathognomonic sign of CD is the "central dot sign", consisting of a small enhancing focus containing a dilated intrahepatic duct with a cystic configuration observed at contrast-enhanced CT and MR (Figure $3 A, B, C$ ). At the histological level, it is related to dense fibrovascular bundles embedding the portal vein and hepatic artery branches, localized around abnormally dilated intrahepatic bile ducts (Figure 3D) (46). Moreover, the initial approach must also include a brain CT scan or MRI to rule out cerebral malformations that could be associated to HRFCD (e.g., Joubert or COACH syndromes) (47-49). During followup, ultrasound with acoustic radiation force impulse elastography may provide a non-invasive tool to stage fibrosis and portal hypertension in children (50).

Liver biopsy can be of help mostly in CHF. Histology may also have a role especially in adults with portal hypertension and chronic liver disease of unknown origin (51). Typical histological findings are thick portal/ peribiliary fibrosis embedding dysgenetic, "exuberant" bile ducts eventually evolving to cystic dilations when CD coexists (Figure $4 A$ ). In CHF, peribiliary fibrosis progresses to porto-portal rather than porto-central bridging, as seen in cirrhosis of more common aetiologies. Another histological lesion strongly suggesting DPM is the persistence of $\mathrm{CD}_{5} 6^{+}$ductal plate remnants (Figure $4 B$ ), together with an increase in hepatic artery branches and hypoplasia or abnormal branching of the portal vein, leading to a picture originally described as "pollard willow" pattern. Of note, these distinctive features are well phenocopied by experimental models, as shown in the Pkbd1-defective mouse (Figure 4C,D).

\section{Treatment}

Clinical management in FLDs is challenging and well- 

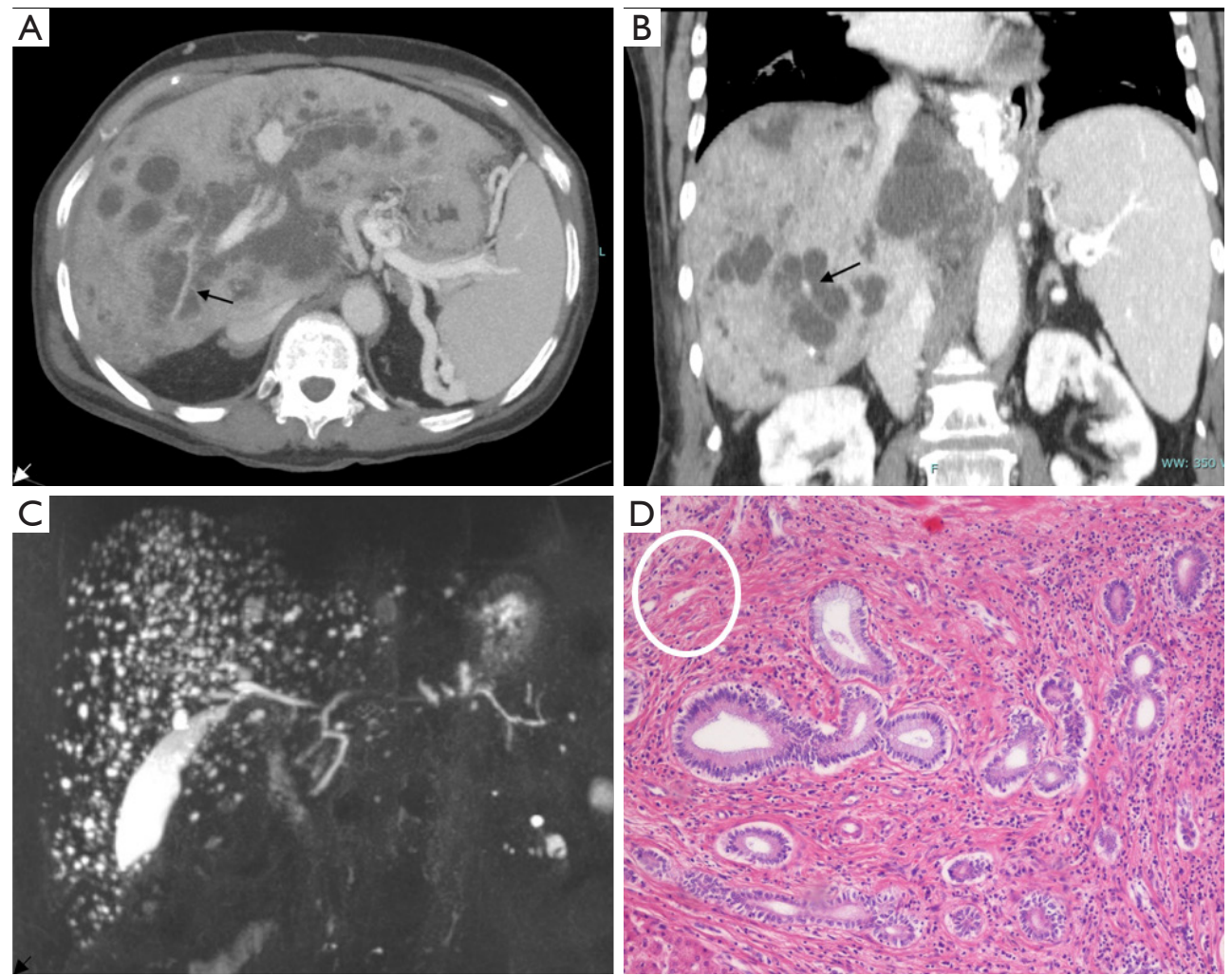

Figure 3 Radiological and histological findings in CD. Axial (A) and coronal (B) reconstruction of 83-year-old woman using contrastenhanced CT. Images outline the diffuse cystic changes of the intrahepatic bile ducts with the typical "central dot sign" (arrows). MR coronal view (C) of a 68-year-old man showing the typical "starry sky" appearance consistent with the presence of multiple microhamartomas. H\&E histological staining $(\times 10)$ (D) show biliary microhamartomas surrounded by a dense stromal reaction composed by fibrotic bundles and inflammatory cells. On the upper left corner (white circle), hepatic artery and portal vein branches are embedded in a dense fibrotic area (histological lesion equivalent of the radiological "central dot sign").

established guidelines are lacking. Thus, a multidisciplinary approach involving hepatologist, nephrologist, radiologist, endoscopist, and surgeon is needed. Liver and renal diseases, when coexisting, progress at different rates and may variably affect the outcome of ongoing treatments. No effective strategies to reverse, or slow disease progression are available in $\mathrm{CHF} / \mathrm{CS} / \mathrm{CD}$, which can be thus considered "orphan" diseases. The treatment is essentially that of the main complications, i.e., portal hypertension, cholangitis and iCCA.

Endoscopic treatment, particularly band ligation, is the current standard of care for primary prophylaxis of bleeding from oesophageal varices and for treatment of bleeding varices, whereas unselective $\beta$-blockers are currently not recommended due to the lack of specific studies in the $\mathrm{CHF} / \mathrm{CS}$ paediatric population (52) and more generally, in the paediatric age. If the bleeding does not respond to endoscopic treatment or in the case of recurrent variceal bleeding, a portal decompressive shunt can be considered in highly specialized hepatological surgical centres, though unusually performed in children and now substantially abandoned in favour of transjugular intrahepatic portosystemic shunt (TIPSS) (53). In the long-term, TIPSS can be a reasonable alternative to surgical shunt, given its feasibility in children. Results from small series are encouraging as they show regression of portal hypertension (oesophageal varices) and reduction in spleen size, with an increase in the platelet count. Of note, TIPSS might delay the time of transplantation, notwithstanding the close monitoring of complications (54). Shunts in HRFCD should be considered with caution if the patient has endstage kidney disease $(45,55)$.

Intrahepatic lithiasis is a common complication of CS/ $\mathrm{CD}$, often associated with bacterial infections responsible 

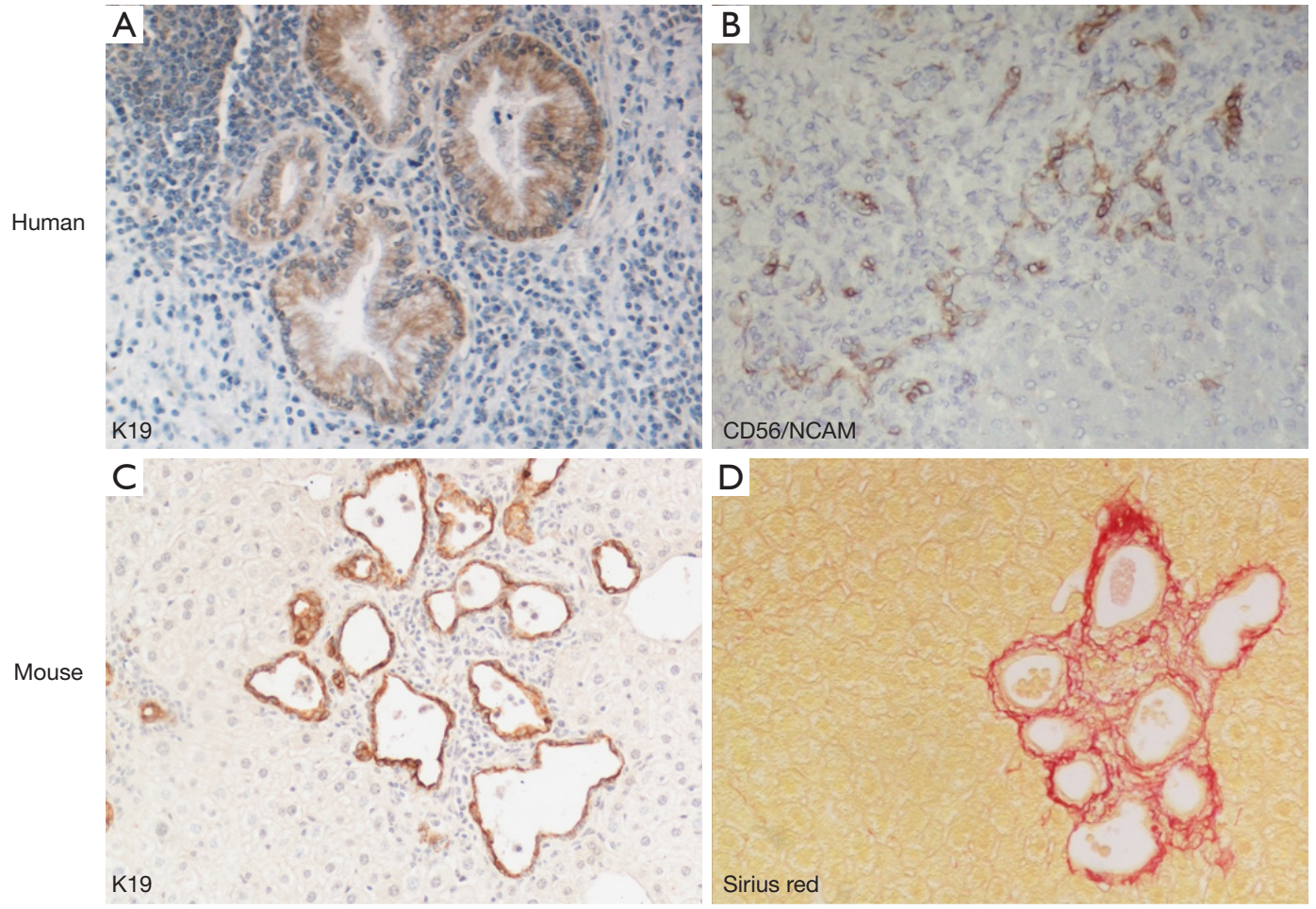

Figure 4 Liver phenotype in FLD, in human and experimental mouse model. Dysgenetic bile ducts immunoreactive for K19 develop a cystlike configuration in enlarged portal tracts containing an abundant inflammatory cell infiltrate (A, $\times 20)$. They are accompanied by ductal plate remnants expressing CD56/NCAM (neural cell adhesion molecule) mostly localized at the portal tract margins $(\mathrm{B}, \times 10)$. These features are phenocopied by the Pkhd1del4/del4 mouse, where microhamartomas and biliary cysts expressing K19 (C, ×10) are tightly surrounded by extensive peribiliary fibrosis confined to the portal area, as shown by histochemical staining for Sirius Red $(\mathrm{D}, \times 10)$.

for recurrent cholangitis, liver abscess and sepsis. In case of high suspicion, antibiotic treatment should be started without hesitation because of the risk of quick deterioration, which is further increased in patients with ARPKD or under immunosuppressive therapy following renal transplantation (56). Ursodeoxycholic acid showed only limited efficacy in reducing the risk of cholangitis or in treating hepatolithiasis (57).

Occurrence of iCCA is a dreadful complication of CHF/ CS/CD, bearing a 100-fold increased risk than the general population (58); more recent small series confirmed a similar prevalence of iCCA in CD/CS, as high as 7\% (59-61). Importantly, in these conditions, iCCA affects young individuals. Unfortunately, given the lack of surveillance programs, the early detection of iCCA is difficult, and thus, eligibility to curative treatments fairly limited (62). There are no specific studies dealing with the treatment of iCCA in the setting of $\mathrm{CHF} / \mathrm{CS} / \mathrm{CD}$. In iCCA in general, surgical resection still represents the only option with curative intent, though feasible only in less than one-third of patients and with a poor 5 -year survival, ranging from $22-44 \%$ $(60,61)$. Since liver transplantation is associated with rapid tumour recurrence and low survival (10-25\%), it is not currently considered in the treatment algorithm of iCCA $(63,64)$. Given the well-established strong chemoresistance of biliary malignancies, systemic and locoregional therapies show scarce efficacy, though targeted and immunotherapy are currently under evaluation (65). Whether genetic alterations amenable of personalized targeted interventions might identify distinctive subgroups of iCCA arising in $\mathrm{CHF} / \mathrm{CD} / \mathrm{CS}$ is yet an unexplored topic.

Surgical resection of hepatic segments affected by intrahepatic bile duct dilation, showed excellent long-term results in selected patients with symptomatic mono-lobar disease without underlying chronic liver disease (66). The largest surgical series-111 patients, $90 \%$ of them with left lobe involvement-reported no peri-surgical mortality and good control of complications by 25 months of median 
follow-up. To maximize the beneficial effects of resection, a thorough preliminary evaluation of the real extension of liver disease is mandatory, since incomplete resection is associated with poor outcome (67). Surgical treatment should be planned as early as possible due to the dual risk of iCCA and infections (as mutually interacting factors) that increases over time (68). In the last few years, endoscopic, radiological and laparoscopic approaches have been used to drain abscesses and clear stones in a less invasive way.

Liver transplantation (LT) remains the only curative option in CHF/CS, with strict indications to patients with bi-lobar involvement, complicated by recurrent cholangitis or portal hypertension (68-70). In the largest published series, collected from the European Transplant Liver Registry and the United Network of Organ Sharing data, survival rates reported were $89 \%, 86 \%, 76 \%$ and $88.5 \%, 81 \%, 78 \%$ at 1,5 , and 10 years, respectively. Predictors of poor outcome were older age and infections at the time of transplant (71). These studies reported a perioperative mortality of $10 \%$, mainly caused by severe infections further facilitated by the immunosuppressive therapy. Therefore, it is recommended to avoid preoperative invasive biliary procedures that can enhance the risk of infections, and to undergo prolonged antibiotic prophylaxis before and after LT.

Another issue is the indication for double liver-kidney transplantation, including its timing. Only a small subset of these patients with HRFCD seem to require double transplantation, either sequentially or in combination. In a large series of 716 HRFCD patients receiving a liver (LT) and/or kidney transplant (KT) between 1990 and 2010, most received KT (86\%), whilst only small numbers received isolated liver transplant $(10 \%)$ or both $(6 \%)$. Moreover, only few patients needed a sequential transplant of the other organ (7\% of LT and $5 \%$ of KT recipients) (72). In the post-transplant setting, it is of utmost importance to preserve the function of the non-transplanted organ still left in place. Therefore, after LT, calcineurin inhibitors must be kept at the lowest effective dose to protect the kidney (73). On the other side, after KT, chronic immunosuppression may favour the development of cholangitis, thus supporting indication to combined $\mathrm{KT}+\mathrm{LT}$ in patients with end-stage renal failure with history of cholangitis or with marked abnormalities of the biliary tree. Furthermore, simultaneous transplant provides the kidney with an immunological advantage, that improves outcome and graft survival in both adults and children (74-76). There are a number of key questions needing consideration by future studies. Since we are dealing with a rare and clinically heterogeneous disease, we must bear in mind that data on LT generated so far have been obtained in patients transplanted for complications related to portal hypertension or recurrent cholangitis rather than for end-stage liver disease, due to the low MELD/PELD typically scored by these patients. Thus, criteria supporting indications to LT/LT + KT lack standardized protocols, making these studies difficult to analyse. Given that the occurrence of an iCCA usually contraindicates liver transplant, it is debated if prophylactic LT could be an option for patients with diffuse bi-lobar disease. A similar issue is a subject of controversy also in patients with primary sclerosing cholangitis $(62,68,77,78)$.

\section{Conclusions}

There is a rising interest in FLDs and it is driven by the important translational implications of genetic cholangiopathies, and the availability of animal and cellular models that phenocopy the disease (29). Deciphering the multiple changes in cell physiology derived from a single ciliary protein defect enable us to gain new insights into the pathophysiology of cholangiopathies. In time, this increased knowledge will translate in innovative therapies, that may be applied beyond these "orphan" diseases, to the more common acquired cholangiopathies and, more broadly, to chronic liver diseases. The lesson from FLDs is indeed prototypical of this concept. Genetic mutations in FPC promote an over-activation of $\beta$-catenin signalling, that triggers an inflammatory response with secretion of chemokines, attraction of macrophages and progressive biliary fibrogenesis. This pathogenetic sequence constitutes a triad, characterized by cyst growth, inflammation and biliary fibrosis, and provides a number of targets for therapeutic intervention. For instance, targeting macrophage infiltration or $\beta$-catenin signalling has led to significant amelioration of portal fibrosis and reduction in biliary cyst expansion in the $P k b d 1$-defective mouse $(10,12,13)$. These approaches, summarized in Table 4, are still limited to experimental conditions, also because of the difficulties in conducting clinical trials in rare diseases. As an example of the important challenges to face in this context, study groups would include small, widely dispersed, mostly paediatric patients, with high phenotypic variability, in absence of validated biomarkers and qualified endpoints (86).

At a more clinical level, there are a number of questions that should be addressed in the next studies. LT represents a valuable therapeutic option, but these patients have low 
Table 4 Experimental therapeutic targets in FLD

\begin{tabular}{|c|c|c|c|}
\hline Target & Mechanism & Agent & Ref. \\
\hline \multirow[t]{2}{*}{ Somatostatin receptors } & \multirow[t]{2}{*}{ Block of cAMP signalling through binding to somatostatin receptors } & Pasireotide ${ }^{1}$ & (79) \\
\hline & & Octreotide $^{1}$ & $(79,80)$ \\
\hline \multirow{2}{*}{\multicolumn{2}{|c|}{$\begin{array}{l}\text { Intracellular } \mathrm{Ca}^{++} \text {levels and toxic bile Block of cAMP signalling by increasing intracellular } \mathrm{Ca}^{++} \\
\text {acids }\end{array}$}} & $\mathrm{UDCA}^{2}$ & (81) \\
\hline & & TRPV4 agonist ${ }^{2}$ & (82) \\
\hline Matrix metalloproteases (MMPs) & Inhibition of MMP function & Marimastat ${ }^{3}$ & (83) \\
\hline \multirow[t]{2}{*}{ PPAR $\gamma$} & \multirow[t]{2}{*}{ Inhibition of ERK1/2 and mTOR-S6 kinase signalling pathways } & Pioglitazione $^{4}$ & (84) \\
\hline & & Telmisartan ${ }^{4}$ & $(84,85)$ \\
\hline Macrophages & Direct inhibition of monocyte-macrophage transdifferentiation & Clodronate $^{5}$ & (31) \\
\hline CXCR3 & Inhibition of monocyte recruitment acting on the CXCL10 receptor & AMG- $487^{5}$ & (30) \\
\hline
\end{tabular}

Adapted from (29). ${ }^{1}$, octreotide and pasireotide demonstrated to slow hepatic cyst growth and to reduce peribiliary fibrosis in PCK rats. Clinical trials in phase I-II are currently ongoing in polycystic liver disease (octreotide in NCT00426153, pasireotide in NCT01670110); ${ }^{2}$, ursodeoxycholic acid (UDCA) and transient receptor potential cation channel subfamily $\vee$ (TRPV4) agonist are strategies under investigation acting both on the same pathways; ${ }^{3}$, marimastat decreased hepatic cystogenesis both in vitro and in PCK rats; ${ }^{4}$, pioglitazone and telmisartan, agonists of peroxisome proliferator-activated receptor- $\gamma$ (PPAR $\gamma$ ), showed efficacy in reducing cyst area, cholangiocyte proliferation and pericystic fibrosis in PCK rats; ${ }^{5}$, bisphosphonate clodronate and AMG-487 were able to reduce either cyst growth or pericystic inflammation and fibrosis in $P$ khd $1^{\text {del//del/4 }}$ mice.

priority compared with candidates with higher MELD/ PELD scores, due to liver decompensation or HCC. These patients might benefit from living-donor LT with consequently shorter waiting times and a lower risk of life-threatening complications $(67,68,87)$. Studies aimed at standardizing access to LT, and at better investigating feasibility and ethical issues about living-donor procedures would be welcome. Finally, the risk of developing CCA is currently one of the major gaps in knowledge especially in children, where cancer is the most frequent cause of late mortality (88). Unfortunately, no standard protocols of surveillance have been produced and therefore, research studies are strongly recommended to clarify the real CCA incidence, long-term follow-up, additional risk factors with the related predictive biomarkers, and the treatment options.

\section{Acknowledgments}

Funding: LF was supported by Progetti di Ricerca di Dipartimento (PRID-DMM) 2017, University of Padua. MS acknowledges the support of the NIH grants DK101528-05 and DK034989, Silvio O. Conte Digestive Diseases Research Core Center (Cellular, Molecular, and Clinical/Translational cores), and a grant from PSC Partners Seeking a Cure Foundation (AWD0002203,
Proposal ID: 18-004707).

\section{Footnote}

Provenance and Peer Review: This article was commissioned by the Guest Editors (Luca Fabris and Mario Strazzabosco) for the series "Recent Advances in Rare Liver Diseases" published in Translational Gastroenterology and Hepatology. The article has undergone external peer review.

Conflicts of Interest: All authors have completed the ICMJE uniform disclosure form (available at http://dx.doi. org/10.21037/tgh-2020-04). The series "Recent Advances in Rare Liver Diseases" was commissioned by the editorial office without any funding or sponsorship. LF and MS served as the unpaid Guest Editors of the series. LF serves as an unpaid editorial board member of Translational Gastroenterology and Hepatology from Sep 2018 to Aug 2020. MS is a member of the advisory board of Esiai/Merk, Bayer, and Engitix, during the conduct of the study. The authors have no other conflicts of interest to declare.

Etbical Statement: The authors are accountable for all aspects of the work in ensuring that questions related to the accuracy or integrity of any part of the work are appropriately investigated and resolved. 
Open Access Statement: This is an Open Access article distributed in accordance with the Creative Commons Attribution-NonCommercial-NoDerivs 4.0 International License (CC BY-NC-ND 4.0), which permits the noncommercial replication and distribution of the article with the strict proviso that no changes or edits are made and the original work is properly cited (including links to both the formal publication through the relevant DOI and the license). See: https://creativecommons.org/licenses/by-nc-nd/4.0/.

\section{References}

1. Desmet VJ. Pathogenesis of Ductal Plate Abnormalities. Mayo Clin Proc 1998;73:80-9.

2. Gunay-Aygun M. Liver and kidney disease in ciliopathies. Am J Med Genet C Semin Med Genet 2009;151C:296306.

3. Gascue C, Katsanis N, Badano JL. Cystic diseases of the kidney: ciliary dysfunction and cystogenic mechanisms. Pediatr Nephrol 2011;26:1181-95.

4. Avidor-Reiss T, Maer AM, Koundakjian E, et al. Decoding cilia function: defining specialized genes required for compartmentalized cilia biogenesis. Cell 2004;117:527-39.

5. Fliegauf M, Benzing T, Omran H. When cilia go bad: cilia defects and ciliopathies. Nat Rev Mol Cell Biol 2007;8:880-93.

6. Mahjoub MR. The importance of a single primary cilium. Organogenesis 2013;9:61-9.

7. Rock N, McLin V. Liver involvement in children with ciliopathies. Clin Res Hepatol Gastroenterol 2014;38:407-14.

8. Adeva M, El-Youssef M, Rossetti S, et al. Clinical and molecular characterization defines a broadened spectrum of autosomal recessive polycystic kidney disease (ARPKD). Medicine (Baltimore) 2006;85:1-21.

9. Rawat D, Kelly DA, Milford DV, et al. Phenotypic variation and long-term outcome in children with congenital hepatic fibrosis. J Pediatr Gastroenterol Nutr 2013;57:161-6.

10. Bergmann C, Küpper F, Dornia C, et al. Algorithm for efficient PKHD1 mutation screening in autosomal recessive polycystic kidney disease (ARPKD). Hum Mutat 2005;25:225-31.

11. Rossetti S, Harris PC. Genotype-phenotype correlations in autosomal dominant and autosomal recessive polycystic kidney disease. J Am Soc Nephrol JASN 2007;18:1374-80.

12. Gunay-Aygun M, Tuchman M, Font-Montgomery E, et al. PKHD1 sequence variations in 78 children and adults with autosomal recessive polycystic kidney disease and congenital hepatic fibrosis. Mol Genet Metab 2010;99:160-73.

13. Available from: http://www.humgen.rwth-aachen.de/index. php?page $=$ database

14. Furu L, Onuchic LF, Gharavi A, et al. Milder presentation of recessive polycystic kidney disease requires presence of amino acid substitution mutations. J Am Soc Nephrol JASN 2003;14:2004-14.

15. Hartung EA, Guay-Woodford LM. Autosomal recessive polycystic kidney disease: a hepatorenal fibrocystic disorder with pleiotropic effects. Pediatrics 2014;134:e833-845.

16. Kaimori JY, Nagasawa Y, Menezes LF, et al. Polyductin undergoes notch-like processing and regulated release from primary cilia. Hum Mol Genet 2007;16:942-56.

17. Menezes LFC, Cai Y, Nagasawa Y, et al. Polyductin, the PKHD1 gene product, comprises isoforms expressed in plasma membrane, primary cilium, and cytoplasm. Kidney Int 2004;66:1345-55.

18. Zhang M-Z, Mai W, Li C, et al. PKHD1 Protein Encoded by the Gene for Autosomal Recessive Polycystic Kidney Disease Associates with Basal Bodies and Primary Cilia in Renal Epithelial Cells. Proc Natl Acad Sci U S A 2004;101:2311-6.

19. Cano DA, Murcia NS, Pazour GJ, et al. Orpk mouse model of polycystic kidney disease reveals essential role of primary cilia in pancreatic tissue organization. Development 2004;131:3457-67.

20. Strazzabosco M, Fabris L. Development of the bile ducts: Essentials for the clinical hepatologist. J Hepatol 2012;56:1159-70.

21. Roskams T, Desmet V. Embryology of extra- and intrahepatic bile ducts, the ductal plate. Anat Rec Hoboken NJ 2007 2008;291:628-35.

22. Santiago I, Loureiro R, Curvo-Semedo L, et al. Congenital cystic lesions of the biliary tree. AJR Am J Roentgenol 2012;198:825-35.

23. Fischer E, Legue E, Doyen A, et al. Defective planar cell polarity in polycystic kidney disease. Nat Genet 2006;38:21-3.

24. Ward CJ, Hogan MC, Rossetti S, et al. The gene mutated in autosomal recessive polycystic kidney disease encodes a large, receptor-like protein. Nat Genet 2002;30:259-69.

25. Germino GG. Linking cilia to Wnts. Nat Genet 2005;37:455-7.

26. Wilson DH, Jarman EJ, Mellin RP, et al. Non-canonical Wnt signalling regulates scarring in biliary disease via the planar cell polarity receptors. Nat Commun 2020;11:445. 
27. Skouloudaki K, Puetz M, Simons M, et al. Scribble participates in Hippo signaling and is required for normal zebrafish pronephros development. Proc Natl Acad Sci U S A 2009; 106:8579-84.

28. Xu D, Lv J, He L, et al. Scribble influences cyst formation in autosomal-dominant polycystic kidney disease by regulating Hippo signaling pathway. FASEB J 2018;32:4394-407.

29. Fabris L, Fiorotto R, Spirli C, et al. Pathobiology of inherited biliary diseases: a roadmap to understand acquired liver diseases. Nat Rev Gastroenterol Hepatol 2019;16:497-511.

30. Kaffe E, Fiorotto R, Pellegrino F, et al. $\beta$-Catenin and interleukin-1 $\beta$-dependent chemokine (C-X-C motif) ligand 10 production drives progression of disease in a mouse model of congenital hepatic fibrosis. Hepatology 2018;67:1903-19.

31. Locatelli L, Cadamuro M, Spirlì C, et al. Macrophage recruitment by fibrocystin-defective biliary epithelial cells promotes portal fibrosis in congenital hepatic fibrosis. Hepatology 2016;63:965-82.

32. Huang G, Brigstock DR. Regulation of hepatic stellate cells by connective tissue growth factor. Front Biosci Landmark Ed 2012;17:2495-507.

33. Jiang L, Fang P, Weemhoff JL, et al. Evidence for a "Pathogenic Triumvirate" in Congenital Hepatic Fibrosis in Autosomal Recessive Polycystic Kidney Disease. BioMed Res Int 2016;2016:4918798

34. Mrug M, Zhou J, Woo Y, et al. Overexpression of innate immune response genes in a model of recessive polycystic kidney disease. Kidney Int 2008;73:63-76.

35. de Tommaso AMA, Santos DSM, Hessel G. Caroli’s disease: 6 case studies. Acta Gastroenterol Latinoam 2003;33:47-51.

36. Umar J, John S. Caroli Disease. StatPearls Publishing; 2019. Available from: http://www.ncbi.nlm.nih.gov/books/ NBK513307/

37. European Association for the Study of the Liver. EASL Clinical Practice Guidelines for the management of patients with decompensated cirrhosis. J Hepatol 2018;69:406-60.

38. Kruis T, Güse-Jaschuck S, Siegmund B, et al. Use of microbiological and patient data for choice of empirical antibiotic therapy in acute cholangitis. BMC Gastroenterol 2020;20:65.

39. Labib PL, Goodchild G, Pereira SP. Molecular Pathogenesis of Cholangiocarcinoma. BMC Cancer 2019;19:185.
40. Srinath A, Shneider BL. Congenital hepatic fibrosis and autosomal recessive polycystic kidney disease. J Pediatr Gastroenterol Nutr 2012;54:580-7.

41. Zerres K, Rudnik-Schöneborn S, Deget F, et al. Autosomal recessive polycystic kidney disease in 115 children: clinical presentation, course and influence of gender. Arbeitsgemeinschaft für Pädiatrische, Nephrologie. Acta Paediatr 1996;85:437-45.

42. Roy S, Dillon MJ, Trompeter RS,et al. Autosomal recessive polycystic kidney disease: long-term outcome of neonatal survivors. Pediatr Nephrol 1997;11:302-6.

43. Guay-Woodford LM, Desmond RA. Autosomal recessive polycystic kidney disease: the clinical experience in North America. Pediatrics 2003;111:1072-80.

44. Bergmann C, Senderek J, Windelen E, et al. Clinical consequences of PKHD1 mutations in 164 patients with autosomal-recessive polycystic kidney disease (ARPKD). Kidney Int 2005;67:829-48.

45. Telega G, Cronin D, Avner ED. New approaches to the autosomal recessive polycystic kidney disease patient with dual kidney-liver complications. Pediatr Transplant 2013;17:328-35.

46. Cannella R, Giambelluca D, Diamarco M, et al. Congenital Cystic Lesions of the Bile Ducts: Imaging-Based Diagnosis. Curr Probl Diagn Radiol 2020;49:285-93.

47. Van De Weghe JC, Rusterholz TDS, Latour B, et al. Mutations in ARMC9, which Encodes a Basal Body Protein, Cause Joubert Syndrome in Humans and Ciliopathy Phenotypes in Zebrafish. Am J Hum Genet 2017;101:23-36.

48. Kumar S, Rankin R. Renal insufficiency is a component of COACH syndrome. Am J Med Genet 1996;61:122-6.

49. Parisi MA. The molecular genetics of Joubert syndrome and related ciliopathies: The challenges of genetic and phenotypic heterogeneity. Transl Sci Rare Dis 2019;4:25-49.

50. Hartung EA, Wen J, Poznick L, et al. Ultrasound Elastography to Quantify Liver Disease Severity in Autosomal Recessive Polycystic Kidney Disease. J Pediatr 2019;209:107-15.e5.

51. Alsomali MI, Yearsley MM, Levin DM, et al. Diagnosis of Congenital Hepatic Fibrosis in Adulthood. Am J Clin Pathol 2020;153:119-25.

52. Büscher R, Büscher AK, Weber S, et al. Clinical manifestations of autosomal recessive polycystic kidney disease (ARPKD): kidney-related and non-kidney-related phenotypes. Pediatr Nephrol 2014;29:1915-25.

53. Shneider BL, Bosch J, de Franchis R, et al. Portal hypertension in children: expert pediatric opinion 
on the report of the Baveno v Consensus Workshop on Methodology of Diagnosis and Therapy in Portal Hypertension. Pediatr Transplant 2012;16:426-37.

54. Verbeeck S, Mekhali D, Cassiman D, et al. Long-term outcome of transjugular intrahepatic portosystemic shunt for portal hypertension in autosomal recessive polycystic kidney disease. Dig Liver Dis 2018;50:707-12.

55. Tsimaratos M, Cloarec S, Roquelaure B, et al. Chronic renal failure and portal hypertension--is portosystemic shunt indicated? Pediatr Nephrol 2000;14:856-8.

56. Guay-Woodford LM, Bissler JJ, Braun MC, et al. Consensus expert recommendations for the diagnosis and management of autosomal recessive polycystic kidney disease: report of an international conference. J Pediatr 2014;165:611-7.

57. Ros E, Navarro S, Bru C, et al. Ursodeoxycholic acid treatment of primary hepatolithiasis in Caroli's syndrome. Lancet 1993;342:404-6.

58. Dayton MT, Longmire WP, Tompkins RK. Caroli's disease: A premalignant condition? Am J Surg 1983;145:41-8.

59. Levy AD, Rohrmann CA, Murakata LA, et al. Caroli's disease: radiologic spectrum with pathologic correlation. AJR Am J Roentgenol 2002;179:1053-7.

60. Bockhorn M, Malagó M, Lang H, et al. The role of surgery in Caroli's disease. J Am Coll Surg 2006;202:928-32.

61. Mabrut JY, Kianmanesh R, Nuzzo G, et al. Surgical management of congenital intrahepatic bile duct dilatation, Caroli's disease and syndrome: long-term results of the French Association of Surgery Multicenter Study. Ann Surg 2013;258:713-21; discussion 721.

62. Jang MH, Lee YJ, Kim H. Intrahepatic cholangiocarcinoma arising in Caroli's disease. Clin Mol Hepatol 2014;20:402-5.

63. Khan SA, Davidson BR, Goldin RD, et al. Guidelines for the diagnosis and treatment of cholangiocarcinoma: an update. Gut 2012;61:1657-69.

64. Rosen CB, Heimbach JK, Gores GJ. Liver transplantation for cholangiocarcinoma. Transpl Int 2010;23:692-7.

65. Banales JM, Cardinale V, Carpino G, et al. Cholangiocarcinoma: current knowledge and future perspectives consensus statement from the European Network for the Study of Cholangiocarcinoma (ENSCCA). Nat Rev Gastroenterol Hepatol 2016;13:261-80.

66. Yamaguchi T, Cristaudi A, Kokudo T, et al. Surgical treatment for monolobular Caroli's disease Report of a 30-year single center case series. Biosci Trends 2018;12:426-31.
67. Mabrut JY, Partensky C, Jaeck D, et al. Congenital intrahepatic bile duct dilatation is a potentially curable disease: long-term results of a multi-institutional study. Ann Surg 2007;246:236-45.

68. Fahrner R, Dennler SGC, Dondorf F, et al. Liver resection and transplantation in Caroli disease and syndrome. J Visc Surg 2019;156:91-5.

69. Lai Q, Lerut J. Proposal for an algorithm for liver transplantation in Caroli's disease and syndrome: putting an uncommon effort into a common task. Clin Transplant 2016;30:3-9.

70. Harring TR, Nguyen NTT, Liu H, et al. Caroli disease patients have excellent survival after liver transplant. J Surg Res 2012;177:365-72.

71. Habib S, Shakil O, Couto OF, et al. Caroli's disease and orthotopic liver transplantation. Liver Transpl 2006;12:416-21.

72. Wen JW, Furth SL, Ruebner RL. Kidney and liver transplantation in children with fibrocystic liver-kidney disease: data from the US Scientific Registry of Transplant Recipients: 1990-2010. Pediatr Transplant 2014;18:726-32.

73. Büscher R, Büscher AK, Cetiner M, et al. Combined liver and kidney transplantation and kidney after liver transplantation in children: Indication, postoperative outcome, and long-term results. Pediatr Transplant 2015;19:858-65.

74. Kitajima K, Ogawa Y, Miki K, et al. Longterm renal allograft survival after sequential liver-kidney transplantation from a single living donor. Liver Transpl 2017;23:315-23.

75. Rogers J, Bueno J, Shapiro R, et al. Results of simultaneous and sequential pediatric liver and kidney transplantation. Transplantation 2001;72:1666-70.

76. de la Cerda F, Jimenez WA, Gjertson DW, et al. Renal graft outcome after combined liver and kidney transplantation in children: UCLA and UNOS experience. Pediatr Transplant 2010;14:459-64.

77. Astarcioglu I, Egeli T, Unek T, et al. Liver Transplant in Patients with Primary Sclerosing Cholangitis: LongTerm Experience of a Single Center. Exp Clin Transplant 2018;16:434-8.

78. Lindor KD, Kowdley KV, Harrison ME. ACG Clinical Guideline: Primary Sclerosing Cholangitis. Am J Gastroenterol 2015;110:646-59.

79. Masyuk TV, Radtke BN, Stroope AJ, et al. Pasireotide is more effective than octreotide in reducing hepatorenal cystogenesis in rodents with polycystic kidney and liver diseases. Hepatology 2013;58:409-21. 
80. Masyuk TV, Masyuk AI, Torres VE, et al. Octreotide inhibits hepatic cystogenesis in a rodent model of polycystic liver disease by reducing cholangiocyte adenosine 3',5'-cyclic monophosphate. Gastroenterology 2007;132:1104-16.

81. Munoz-Garrido P, Marin JJG, Perugorria MJ, et al. Ursodeoxycholic acid inhibits hepatic cystogenesis in experimental models of polycystic liver disease. J Hepatol 2015;63:952-61.

82. Gradilone SA, Masyuk TV, Huang BQ, et al. Activation of Trpv4 reduces the hyperproliferative phenotype of cystic cholangiocytes from an animal model of ARPKD. Gastroenterology 2010;139:304-14.e2.

83. Urribarri AD, Munoz-Garrido P, Perugorria MJ, et al. Inhibition of metalloprotease hyperactivity in cystic cholangiocytes halts the development of polycystic liver diseases. Gut 2014;63:1658-67.

84. Yoshihara D, Kurahashi H, Morita M, et al. PPAR-

doi: 10.21037/tgh-2020-04

Cite this article as: Lasagni A, Cadamuro M, Morana G, Fabris L, Strazzabosco M. Fibrocystic liver disease: novel concepts and translational perspectives. Transl Gastroenterol Hepatol 2021;6:26. gamma agonist ameliorates kidney and liver disease in an orthologous rat model of human autosomal recessive polycystic kidney disease. Am J Physiol Renal Physiol 2011;300:F465-74.

85. Yoshihara D, Kugita M, Sasaki M, et al. Telmisartan ameliorates fibrocystic liver disease in an orthologous rat model of human autosomal recessive polycystic kidney disease. PLoS One 2013;8:e81480.

86. Kempf L, Goldsmith JC, Temple R. Challenges of developing and conducting clinical trials in rare disorders. Am J Med Genet A 2018;176:773-83.

87. Takatsuki M, Uemoto S, Inomata Y, et al. Living-donor liver transplantation for Caroli's disease with intrahepatic adenocarcinoma. J Hepatobiliary Pancreat Surg 2001;8:284-6.

88. Soares KC, Goldstein SD, Ghaseb MA, et al. Pediatric choledochal cysts: diagnosis and current management. Pediatr Surg Int 2017;33:637-50. 